Publications of the Astronomical Society of the Pacific, ACCEPTED

Preprint typeset using $\mathrm{LT}_{\mathrm{E}} \mathrm{X}$ style emulateapj v. 10/10/03

\title{
THE PRODUCTION RATE AND EMPLOYMENT OF PH.D. ASTRONOMERS
}

\author{
TRAVIS S. MetCALFE \\ High Altitude Observatory and Scientific Computing Division, \\ National Center for Atmospheric Research, P.O. Box 3000, Boulder, CO 80307 USA \\ Publications of the Astronomical Society of the Pacific, ACCEPTED
}

\begin{abstract}
In an effort to encourage self-regulation of the astronomy job market, I examine the supply of, and demand for, astronomers over time. On the supply side, I document the production rate of Ph.D. astronomers from 1970 to 2006 using the UMI Dissertation Abstracts database, along with data from other independent sources. I compare the long-term trends in Ph.D. production with federal astronomy research funding over the same time period, and I demonstrate that additional funding is correlated with higher subsequent Ph.D. production. On the demand side, I monitor the changing patterns of employment using statistics about the number and types of jobs advertised in the AAS Job Register from 1984 to 2006. Finally, I assess the sustainability of the job market by normalizing this demand by the annual Ph.D. production. The most recent data suggest that there are now annual advertisements for about one postdoctoral job, half a faculty job, and half a research/support position for every new domestic Ph.D. recipient in astronomy and astrophysics. The average new astronomer might expect to hold up to 3 jobs before finding a steady position.
\end{abstract}

Subject headings: sociology of astronomy

\section{BACKGROUND}

From 1982 to 1992, federal funding for astronomy research increased by more than $80 \%$, fueled primarily by NASA funding related to the Hubble Space Telescope. Trailing this trend by several years, the annual production of new Ph.D. astronomers more than doubled. Meanwhile, the total number of jobs advertised in the American Astronomical Society (AAS) Job Register remained fairly constant. Responsible undergraduate programs began to lecture incoming astronomy majors about this imbalance in the job market, comparing the statistical odds of a long-term career in astronomy to the chances of becoming a professional athlete. The faculty in some graduate programs started to debate the idea of limiting the number of incoming students ("birth control"), and they made efforts to track and publicize the long-term career progress of their Ph.D. recipients (e.g., see Dinerstein 1996).

It was in this atmosphere that Thronson (1991) devised a model to describe the surplus production of new astronomers. He observed that overproduction appears to be built into the system, making the mathematical formulation of the problem similar to that of industrial pollution-an unintended side effect of the process. He calculated the future astronomer surplus under a variety of possible circumstances, including expected retirement rates and increased research funding. The most likely of the scenarios predicted a short-lived reduction in the astronomer surplus over a decade or so, followed by continued growth in the overproduction rate.

A few years later, Harris (1994) identified some reliable sources of data to evaluate the astronomy job market over time. He looked at the number of dissertations published in the subject area of astronomy and astrophysics using the UMI Dissertation Abstracts database (UMI) to track new Ph.D. recipients. He noted that the American Institute of Physics (AIP) tabulates the number of degrees awarded by astronomy departments annually, but astronomers who receive their degrees from physics departments have not been tracked until the past few years. To quantify the number of long-term jobs

Electronic address: travis@hao.ucar.edu available each year, Harris examined advertisements in the AAS Job Register. This has some inherent limitations, but it is still the best source available.

Thanks to the Internet, most of these data sources are now much more accessible, and we can easily examine the trends in $\mathrm{Ph} . \mathrm{D}$. production and job availability over an extended time baseline. Further data on the trends in astronomy research funding are also available, allowing us to investigate the possible connection with Ph.D. production predicted by Thronson (1991). In section 2, I document the sources of these data for future reference and I present tables that can be updated annually as new statistics become available. I examine the historical trends of both the supply and demand in section 3 . and I discuss the results in section 4

\section{DATA SOURCES}

The Statistical Research Center of the AIP has been surveying every astronomy Ph.D. program in the U.S. since 1972. An archive of their annual reports beginning in 1980 is available online 1 , and the most recent "Roster of Astronomy Departments" (Nicholson \& Mulvey 2007) appears on a separate page $^{2}$. I received the complete set of data on astronomy Ph.D. production from AIP staff (P. Mulvey, personal communication), which is listed under the heading "AIP" in Table1 These totals do not include degrees that were conferred by physics departments to Ph.D. recipients who complete a dissertation in a subject related to astrophysics, even though this is the most popular subfield among domestic first year physics graduate students (Mulvey \& Tesfaye 2006).

The NSF Division of Science Resources Statistics has documented the annual number of astronomy and astrophysics Ph.D. recipients through the Survey of Earned Doctorates (SED), a voluntary program administered by graduate deans at accredited universities. Over the past decade, more than $90 \%$ of new doctorates have participated in this survey-at many institutions, it is now a requirement for graduation. Historical data from this survey were compiled in a recent NSF

\footnotetext{
1 http://aip.org/statistics/trends/archives/astrorost.htm

2 http://aip.org/statistics/trends/gradtrends.html
} 
TABLE 1

Ph.D. Production AND Research Funding.

\begin{tabular}{rrrrrrrr}
\hline \hline \multicolumn{6}{c}{ Ph.D.s } & \multicolumn{4}{c}{ Research Funding (M\$) } \\
Year & AIP & SED & UMI & Total & NASA & NSF \\
\hline 1970 & $\ldots$ & 111 & 141 & 136.455 & 100.875 & 19.922 \\
1971 & $\ldots$ & 113 & 119 & 126.852 & 90.688 & 23.236 \\
1972 & 75 & 129 & 173 & 136.405 & 89.859 & 25.608 \\
1973 & 80 & 131 & 147 & 123.085 & 82.020 & 27.080 \\
1974 & 97 & 133 & 172 & 136.030 & 91.564 & 30.283 \\
1975 & 76 & 131 & 162 & 168.319 & 122.002 & 29.093 \\
1976 & 93 & 150 & 186 & 163.093 & 116.575 & 31.754 \\
1977 & 96 & 120 & 164 & 197.305 & 140.702 & 41.585 \\
1978 & 93 & 138 & 173 & 213.601 & 148.603 & 46.614 \\
1979 & 90 & 115 & 152 & 284.429 & 213.272 & 51.064 \\
1980 & 106 & 121 & 133 & 285.671 & 229.724 & 34.512 \\
1981 & 82 & 109 & 144 & 281.027 & 198.674 & 59.146 \\
1982 & 70 & 102 & 121 & 275.784 & 184.630 & 65.411 \\
1983 & 81 & 115 & 129 & 357.416 & 265.687 & 64.638 \\
1984 & 74 & 98 & 120 & 382.292 & 277.013 & 77.534 \\
1985 & 66 & 100 & 150 & 414.743 & 310.668 & 76.554 \\
1986 & 86 & 109 & 132 & 468.160 & 362.547 & 77.808 \\
1987 & 72 & 100 & 166 & 522.187 & 410.651 & 76.789 \\
1988 & 94 & 130 & 171 & 470.838 & 364.730 & 77.638 \\
1989 & 94 & 113 & 223 & 541.939 & 428.963 & 83.373 \\
1990 & 89 & 128 & 240 & 597.154 & 462.320 & 104.613 \\
1991 & 73 & 125 & 233 & 631.657 & 501.766 & 101.712 \\
1992 & 93 & 134 & 254 & 739.057 & 584.504 & 116.126 \\
1993 & 119 & 145 & 256 & 686.281 & 520.184 & 109.711 \\
1994 & 117 & 144 & 271 & 747.590 & 562.962 & 122.065 \\
1995 & 133 & 173 & 321 & 764.458 & 593.089 & 126.225 \\
1996 & 126 & 192 & 303 & 728.958 & 570.450 & 119.946 \\
1997 & 117 & 198 & 320 & 774.740 & 621.324 & 115.212 \\
1998 & 116 & 206 & 310 & 732.150 & 575.093 & 113.248 \\
1999 & 88 & 159 & 267 & 757.859 & 578.973 & 124.395 \\
2000 & 139 & 185 & 291 & 880.267 & 710.006 & 130.702 \\
2001 & 101 & 186 & 253 & 759.195 & 559.539 & 153.919 \\
2002 & 102 & 141 & 251 & 751.527 & 540.243 & 167.921 \\
2003 & 88 & 167 & 254 & 871.757 & 639.761 & 192.179 \\
2004 & 116 & 165 & 297 & 920.763 & 678.304 & 202.092 \\
2005 & 91 & 186 & 269 & & $\ldots$ & $\ldots$ & $\ldots$ \\
2006 & 119 & $\ldots$ & 284 & & $\ldots$ & $\ldots$ & $\ldots$ \\
\hline \hline & & & & & &
\end{tabular}

report (Thurgood et al. 2006), while data from the past decade appeared in a separate publication (Hill 2007). The data from 1970 to 2005, which include degrees conferred by both astronomy and physics departments, are listed under the heading "SED" in Table 1,

Although the SED data represent a substantially complete survey, they do not include dissertations that are relevant to astronomy and astrophysics but were completed in another primary field. Following Harris (1994), I used the online version of the UMI Dissertation Abstracts database ${ }^{3}$ to obtain an independent estimate of the number of dissertations related to astronomy and astrophysics that were published annually from 1970 to 2006. For each calendar year, I queried the "Dissertations \& Theses" database for doctoral dissertations with a subject code of astronomy or astrophysics that were written in English and awarded a Ph.D. by a domestic university. The results are shown in Table 1 under the heading "UMI". Since these queries included dissertations with primary subject codes in other fields (e.g., mathematics or chemistry) the

\footnotetext{
${ }^{3}$ http://proquest.umi.com/login
}

TABLE 2

Number of AAS Job Register Ads.

\begin{tabular}{rrrrrrrrrr}
\hline \hline Year & All & PV & TT & NT & \multicolumn{2}{c}{ R } & RS & MO & F \\
\hline 1984 & 275 & 77 & 66 & $\cdots$ & 72 & $\cdots$ & $\ldots$ & $\cdots$ \\
1985 & 338 & 149 & 81 & $\ldots$ & 118 & $\ldots$ & $\ldots$ & $\ldots$ \\
1986 & 257 & $\ldots$ & $\ldots$ & $\ldots$ & $\ldots$ & $\ldots$ & $\ldots$ & $\ldots$ \\
1987 & 240 & $\ldots$ & $\ldots$ & $\ldots$ & $\ldots$ & $\ldots$ & $\ldots$ & $\ldots$ \\
1988 & 321 & 157 & $\ldots$ & $\ldots$ & $\ldots$ & $\ldots$ & $\ldots$ & $\ldots$ \\
1989 & 351 & $\ldots$ & $\ldots$ & $\ldots$ & $\ldots$ & $\ldots$ & $\ldots$ & $\ldots$ \\
1990 & 240 & 120 & 32 & $\ldots$ & $\ldots$ & $\ldots$ & $\ldots$ & $\ldots$ \\
1991 & 304 & 137 & 30 & $\ldots$ & $\ldots$ & $\ldots$ & $\ldots$ & $\ldots$ \\
1992 & 327 & 127 & 40 & 13 & 63 & 57 & 21 & 0.140 \\
1993 & 315 & 66 & 59 & 12 & 42 & 60 & 23 & 0.160 \\
1994 & 318 & 140 & 44 & 15 & 14 & 76 & 24 & 0.233 \\
1995 & 425 & 200 & 54 & 23 & 73 & 52 & 17 & 0.268 \\
1996 & 426 & 210 & 63 & 15 & 65 & 56 & 15 & 0.211 \\
1997 & 469 & 208 & 74 & 9 & 55 & 49 & 65 & 0.230 \\
1998 & 569 & 224 & 77 & 23 & 112 & 68 & 51 & 0.210 \\
1999 & 623 & 206 & 94 & 43 & 130 & 38 & 67 & 0.230 \\
2000 & 739 & 277 & 108 & 39 & 132 & 66 & 63 & 0.264 \\
2001 & 715 & 310 & 93 & 59 & 93 & 56 & 78 & 0.288 \\
2002 & 716 & 370 & 117 & 29 & 109 & 3 & 46 & 0.198 \\
2003 & 722 & 389 & 119 & 22 & 78 & 29 & 85 & 0.072 \\
2004 & 714 & 333 & 113 & 22 & 117 & 28 & 101 & 0.251 \\
2005 & 673 & 334 & 111 & 14 & 88 & 19 & 102 & 0.276 \\
2006 & 738 & 388 & 129 & 22 & 87 & 19 & 93 & 0.276 \\
\hline \hline
\end{tabular}

net effect is to slightly inflate the supply of Ph.D. recipients, leading to a worst case scenario for job seekers. While the normalization of job market numbers to assess sustainability may be sensitive to the choice of degree data, the overall trends should be similar.

In addition to degree data, the NSF Division of Science Resources Statistics also documents the trends in federal funding for research and development. One of their recent reports provides a breakdown of federal funding from 1970 to 2003 by both agency and the detailed field of science and engineering (Meeks 2004). I obtained data for the most recent years from several additional NSF reports (Meeks 2005, 2006; Jankowski 2007). Table 1 includes the total research funding, along with the amounts contributed by NASA and the NSF, reported as the actual dollar values (in millions) for each year ${ }^{4}$. To correct these values for inflation, I used the composite outlay deflators for "other grants" from the FY 2006 U.S. budget", which normalizes all values to constant FY 2000 dollars. Unfortunately, even the historical values of the inflation indices adopted by the government appear to change slightly from one budget to the next. So, any future updates to the research funding data in Table 1 should re-adjust the historical numbers using deflators from the most recent federal budget.

The total number of jobs advertised in the Job Register from 1984 to 2006 is available from the AAS Annual Reports, published each year in the BAAS (e.g., Marvel 2007). A detailed breakdown by advertiser-specified job type has been compiled consistently since 1992, including the following categories: postdoctoral and visitor (PV), tenure-track (TT), non-tenuretrack $(\mathrm{NT})$, research $(\mathrm{R})$, research support (RS), management

\footnotetext{
${ }^{4}$ The specific programs included in these totals were determined by each agency in their response to the annual Survey of Federal Funds for Research and Development

${ }^{5}$ http://gpoaccess.gov/usbudget/fy06/sheets/hist10z1.xls
} 


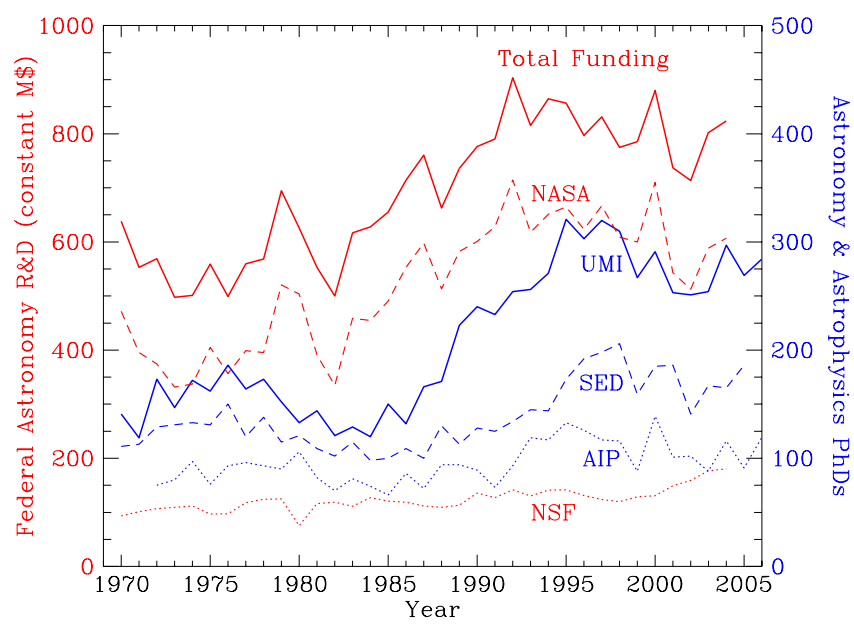

FIG. 1.- Left axis: Total federal funding for astronomy research and development in millions of FY 2000 dollars (upper solid line), including the annual totals from NASA (upper dashed line) and the NSF (lower dotted line). Right axis: Historical Ph.D. production from AIP surveys of astronomy departments (upper dotted line), from the NSF Survey of Earned Doctorates (SED; lower dashed line) and from all dissertations included in the UMI Dissertation Abstracts database and categorized in the subject area of astronomy and astrophysics (lower solid line).

and other (MO). The available data for each of these categories are listed in Table 2. Note that the total number of jobs advertised in each year (shown under the heading "All") is not exactly equal to the sum of the six categories listed. The total also includes jobs categorized as predoctoral, which are omitted from Table 2 because they are not relevant to this study. Statistics about the type of institution offering the jobs are also compiled, including whether it is in a foreign country. To evaluate this contribution to the demand for astronomers, the final column of Table 2 lists the fraction of jobs that were classified as foreign $(\mathrm{F})$. The primary limitation of the Job Register data is that they provide no way of evaluating which advertised positions are new, i.e. which jobs arise from retirement or death as opposed to faculty shuffle. This limitation is generally not relevant to shorter-term postdoctoral jobs, and assuming a reasonably constant number of faculty shuffles over time it will affect the normalization but not the trends in longer-term positions. This may partially offset the impact of adopting the slightly inflated degree data from UMI.

\section{RESULTS}

Using these data, drawn from reliable and accessible sources that are likely to be updated annually, we can evaluate the current state of the astronomy job market and how it has changed over time.

\subsection{Supply of Astronomers}

The data from Table 1 on the supply of astronomers, and the inflation-corrected federal research funding that might be one of its primary drivers, are shown in Figure 1. The left axis shows funding levels in millions of FY 2000 dollars for the total federal investment (upper solid line), and for the contributions from NASA (upper dashed line) and the NSF (lower dotted line). The most striking thing about these data is how NASA dominates the total federal funding for astronomy research, and how it is responsible for nearly all of the volatility. By contrast, astronomy funding from the NSF has shown fairly steady growth, nearly doubling its real value over the

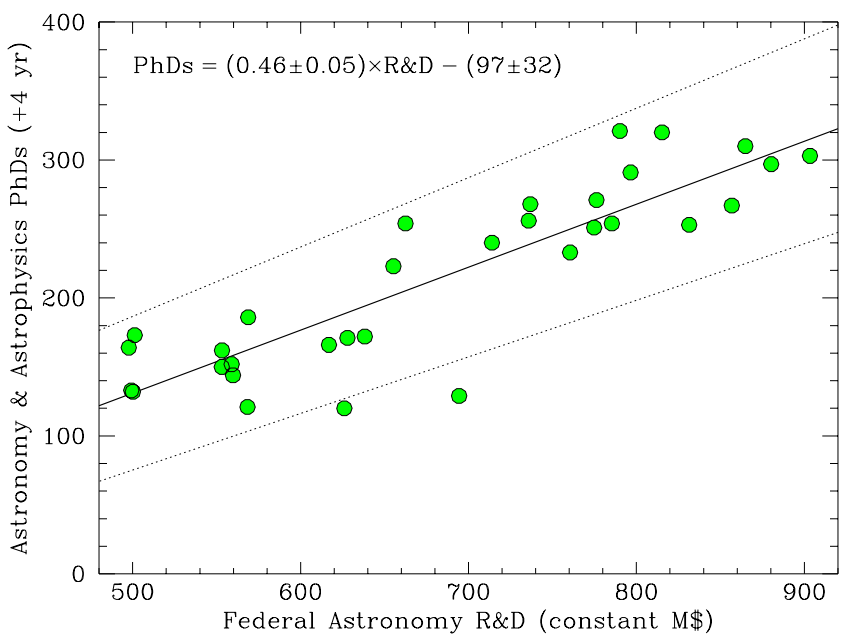

FIG. 2.- The correlation between inflation-adjusted federal research funding and $\mathrm{Ph} . \mathrm{D}$. production related to astronomy and astrophysics 4 years later. The best linear fit (solid line) suggests that an increase of $\$ 100$ million in federal funding ultimately leads to the production of 40-50 additional Ph.D. recipients.

last 35 years. Between 1982 and 1992, NASA funding for astronomy more than doubled. This expansion was driven primarily by preparations for the Hubble Space Telescope, but also by the Compton Gamma Ray Observatory and other missions (E. Suckow, personal communication).

The right axis of Figure 1 shows the total number of Ph.D. recipients each year in astronomy and astrophysics from UMI (lower solid line) and SED (lower dashed line), as well as the subset of those coming from pure astronomy departments (upper dotted line). The correlation between the trend in Ph.D. recipients and the trend in federal funding is apparent, though there may be a delay of several years between the two curves. Degrees from astronomy departments account for about $25 \%$ of the observed increase in Ph.D. recipients between the mid1980's and mid-1990's, while most of the new degrees came from other departments.

To examine the relationship between federal funding and Ph.D. production more quantitatively, Figure 2 shows the correlation between inflation-adjusted research funding in a given year and the number of new degrees related to astronomy and astrophysics 4 years later (from UMI). Similar correlations were produced for time delays ranging from 0 to 5 years, but a 4 year delay minimized the fractional errors on the fit parameters (formally, all of the fits were statistically equivalent). The best linear fit is shown as a solid line, with the $\pm 1 \sigma$ limits on the fit parameters indicated with dotted lines. The correlation suggests that an incremental increase of $\$ 100$ million in federal funding may lead to the production of 40-50 additional $\mathrm{Ph}$.D. recipients in subsequent years (4 years is the median time to Ph.D. after the initial 2 years of coursework).

\subsection{Demand for Astronomers}

The data from Table 2 on the demand for astronomers from 1984 to 2006 are shown in Figure 3 The total number of jobs advertised (upper solid line) is shown along with the numbers of postdoctoral and visitor positions (dotted line), research and research support jobs (dashed line), as well as tenure-track (lower solid line), non-tenure-track (long dashed line), management and other (dash-dot line) advertisements. The growth in the total number of jobs advertised through- 


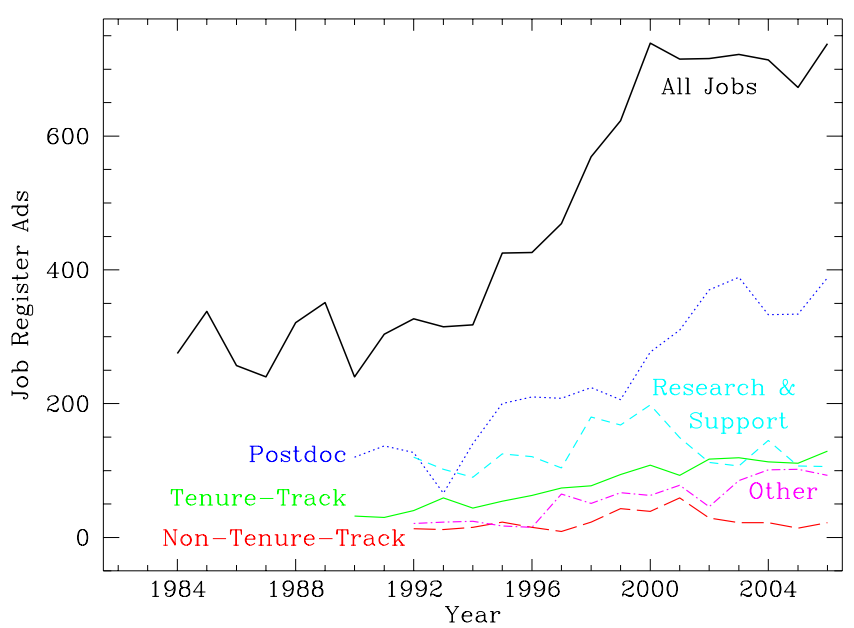

FIG. 3. - The number of jobs advertised in the AAS Job Register from 1984 to 2006 (upper solid line). A detailed breakdown by advertiser-specified job type is available beginning in the early 1990's, and is shown for positions categorized as postdoctoral and visitor (dotted line), research and research support (dashed line), tenure-track (lower solid line), non-tenure-track (long dashed line), along with management and other positions (dot-dashed line).

out the 1990's is remarkable, and is dominated by an increase in the number of postdoctoral positions, though all job categories experienced some growth during this period. The most recent data show some anti-correlated trends after the year 2000, suggesting a possible shift from research and support positions to postdocs, and from non-tenure-track to tenuretrack jobs.

To assess the relative sustainability of the job market, we can normalize these job advertisements by the number of new Ph.D. recipients in each year (from UMI). These data are shown in Figure 4 for all advertised jobs (upper solid line), postdoctoral and visitor positions (dotted line), research and research support jobs (dashed line), tenure-track and nontenure-track positions (lower solid line), as well as management and other advertisements (dash-dot line). By normalizing in this way, the vertical axis can be thought of as a relative probability of obtaining a given type of position for each class of Ph.D. recipients. It is not exactly a probability, since the number of foreign $\mathrm{Ph}$.D. recipients competing for some jobs may exceed the number of foreign jobs advertised, and because some Ph.D. recipients from previous years will be competing for some jobs while others will eventually choose to work outside the field or in a foreign country.

There now appears to be more than one postdoctoral position advertised for every new domestic $\mathrm{Ph}$.D. recipient related to astronomy and astrophysics. This surge in the relative probability of obtaining a postdoc position can be traced to both a slightly declining population of new Ph.D. recipients since a peak in the mid-1990's (Figure 1), as well as the larger number of postdoc positions advertised-possibly at the expense of longer-term research jobs (Figure 3 ). At best, half of all recent $\mathrm{Ph}$.D. recipients might expect to obtain a faculty position, while the other half will likely end up in a research or support position. While this situation may not be ideal for job seekers who prefer to remain in academia, it was much more competitive a decade ago with nearly 6 new Ph.D. astronomers in 1995 for every new tenure-track job. If we assume that all advertised jobs are eventually filled by someone in a related field, then the upper solid line in Figure 4 suggests that the

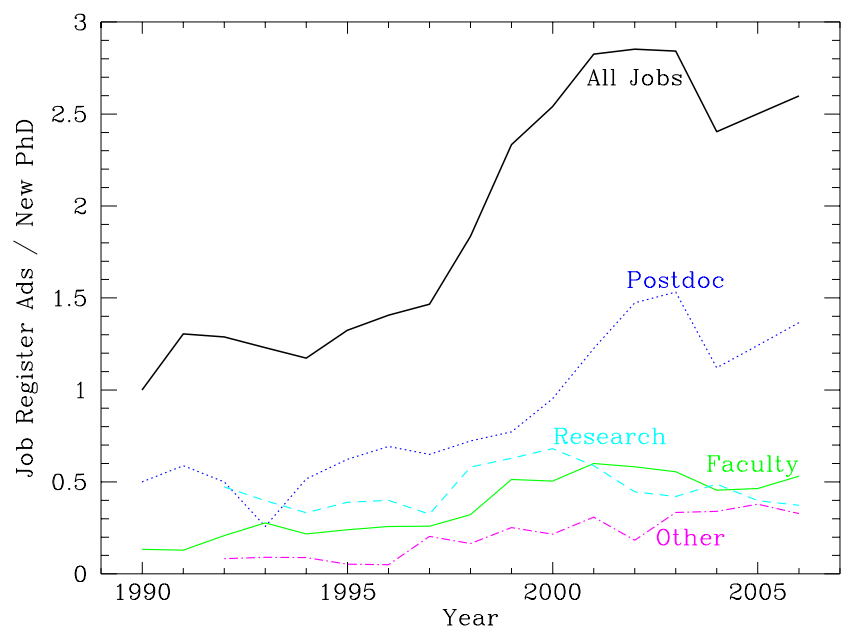

FIG. 4. - Job advertisements in several categories, normalized by the UMI measure of astronomy and astrophysics Ph.D. production from 1990 to 2006. There is now more than one postdoctoral or visitor position (dotted line), about half a tenure-track or non-tenure-track job (lower solid line) and half a research or research support position (dashed line) advertised for each new Ph.D. recipient. If all advertised jobs (upper solid line) are filled each year, then the average new Ph.D. might expect to hold between 2 and 3 of them before finding a stable position.

average new Ph.D. might expect to hold between 2 and 3 jobs before finding a more permanent position.

\section{DISCUSSION}

We are now in a position to evaluate the predictions of Thronson (1991), who made 20 year projections of several models for the production of astronomers over time. His definition of the "astronomer surplus" was the annual ratio of new astronomy Ph.D. recipients tabulated by the AIP relative to new tenure-track faculty positions advertised in the AAS Job Register, which he calculated to be around 2.5 in 1991. Regardless of the absolute level of overproduction, his most realistic models included the competing effects of increased research funding (which leads to growth in the surplus), and retirement (which briefly absorbs some of the surplus). These models predicted a gradual decline in the astronomer surplus over about 10 years, followed by continued overproduction: "A momentary abundance of jobs will be subsequently compensated for by training of new graduate students by a new cohort of young professors". Indeed, the anticipated impact of increased research funding on Ph.D. production has now been quantified in Figure 2] Furthermore, the slowly evolving ratio of faculty positions to new Ph.D. recipients in Figure 4 shows exactly the predicted behavior-peaking in 2001, and gradually leveling off or declining in more recent years.

Despite the accuracy of Thronson's predictions, a broader definition of sustainability may now be necessary, since the character of the astronomy job market appears to have shifted in the meantime. Astronomy research is increasingly moving towards automation and large collaborations where service positions are becoming more important (Sage 2001). This cultural shift might be reflected in the surge of new research and support positions between 1997 and 2002, which may have absorbed some of the astronomer surplus generated during the more competitive conditions of the early 1990's. More recently, as the new cadre of young faculty have attempted to recruit a limited supply of graduate students, some may 
have turned to postdocs to maintain their research productivity. This could be responsible for the unprecedented expansion of postdoctoral jobs after 1999-but as startup funds are depleted and external research funding stays relatively constant, these "holding pattern" positions could evaporate. The most recent data show the first signs of a possible slowdown.

The most important aspect of the ongoing cultural shift in the astronomy job market is the persistent gap between expectations and reality. When first year graduate students are surveyed by the AIP, fully $87 \%$ of those in astronomy departments say they would like to end up in an academic position, while only $8 \%$ express a desire to work in a national lab or research position (Mulvey \& Tesfaye 2006). By contrast, Figure 4 suggests that less than $50 \%$ will ultimately obtain academic positions - and probably fewer, since the turnover at universities appears to be episodic. Graduate programs in astronomy should prepare their students for this reality.

Having documented the historical trends in the astronomy job market and the forces that help shape it, it should be straightforward to update the analysis periodically as new data emerge. In the future, I hope to make such updates available through the Career Services section of the AAS website ${ }^{6}$, and disseminate them through astro-ph. With such information readily available, incoming graduate students can make informed decisions about their long-term career prospects, and faculty can nurture appropriate skills in the next generation of astronomers.

This work was motivated by my involvement with the AAS Employment Committee, which is charged with facilitating the professional development and employment of astronomers at all career stages and on all career paths, and promoting balance and fairness in the job market. The National Center for Atmospheric Research is a federally funded research and development center sponsored by the National Science Foundation.

\footnotetext{
${ }^{6}$ http://aas.org/career/
}

\section{REFERENCES}

Dinerstein, H. 1996, BAAS, 28, 1277

Harris, H. C. 1994, ASP Conf., 57, 12

Hill, S. T. 2007, NSF Report 07-305, (www.nsf.gov/statistics/nsf07305/)

Jankowski, J. E. 2007, NSF Report 07-323, (www.nsf.gov/statistics/ nsf07323/)

Marvel, K. 2007, BAAS, 39, 283

Meeks, R. L. 2004, NSF Report 04-335, (www.nsf.gov/statistics/nsf04335/)

Meeks, R. L. 2005, NSF Report 05-307, (www.nsf.gov/statistics/nsf05307/)

Meeks, R. L. 2006, NSF Report 06-313, (www.nsf.gov/statistics/nsf06313/)
Mulvey, P. J. \& Tesfaye, C. L. 2006, AIP Report R-207.35

Mulvey, P. 2007, personal communication

Sage, L. 2001, Nature, 414, 4

Suckow, E. 2007, personal communication

Thronson, H. A., Jr. 1991, PASP, 103, 90

Thurgood, L., Golladay, M. J. \& Hill, S. T. 2006, NSF Report 06-319, (www.nsf.gov/statistics/nsf06319/) 